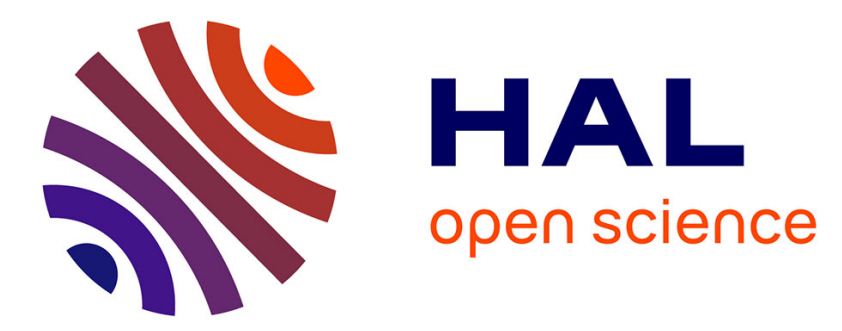

\title{
Load carrying capacity of systems within a global safety perspective Part II. Attractor/basin integrity under dynamic excitations
}

\author{
Stefano Lenci, Giuseppe Rega
}

\section{- To cite this version:}

Stefano Lenci, Giuseppe Rega. Load carrying capacity of systems within a global safety perspective Part II. Attractor/basin integrity under dynamic excitations. International Journal of Non-Linear Mechanics, 2011, 46 (9), pp.1240. 10.1016/j.ijnonlinmec.2011.05.021 . hal-00784917

\section{HAL Id: hal-00784917 \\ https://hal.science/hal-00784917}

Submitted on 5 Feb 2013

HAL is a multi-disciplinary open access archive for the deposit and dissemination of scientific research documents, whether they are published or not. The documents may come from teaching and research institutions in France or abroad, or from public or private research centers.
L'archive ouverte pluridisciplinaire $\mathbf{H A L}$, est destinée au dépôt et à la diffusion de documents scientifiques de niveau recherche, publiés ou non, émanant des établissements d'enseignement et de recherche français ou étrangers, des laboratoires publics ou privés. 


\section{Author's Accepted Manuscript}

Load carrying capacity of systems within a global safety perspective Part II. Attractor/basin integrity under dynamic excitations

Stefano Lenci, Giuseppe Rega

PII: $\quad$ S0020-7462(11)00150-8

DOI: doi:10.1016/j.ijnonlinmec.2011.05.021

Reference: NLM1892

To appear in: International Journal of Non-

Linear Mechanics

Received date: 20 February 2011

Revised date: 26 April 2011

Accepted date: 7 May 2011

Cite this article as: Stefano Lenci and Giuseppe Rega, Load carrying capacity of systems within a global safety perspective Part II. Attractor/basin integrity under dynamic excitations, International Journal of Non-Linear Mechanics, doi:10.1016/j.ijnonlinmec.2011.05.021

This is a PDF file of an unedited manuscript that has been accepted for publication. As a service to our customers we are providing this early version of the manuscript. The manuscript will undergo copyediting, typesetting, and review of the resulting galley proof before it is published in its final citable form. Please note that during the production process errors may be discovered which could affect the content, and all legal disclaimers that apply to the journal pertain. 


\title{
LOAD CARRYING CAPACITY OF SYSTEMS WITHIN A GLOBAL SAFETY PERSPECTIVE. PART II. ATTRACTOR/BASIN INTEGRITY UNDER DYNAMIC EXCITATIONS
}

\author{
Stefano Lenci \\ Department of Architecture, Buildings and Structures \\ Polytechnic University of Marche, Ancona, Italy, lenci@univpm.it \\ Giuseppe Rega \\ Department of Structural and Geotechnical Engineering \\ Sapienza University of Rome, Rome, Italy, Giuseppe.Rega@uniroma1.it
}

\begin{abstract}
The effects of the dynamic excitation on the load carrying capacity of mechanical systems are investigated with reference to the archetypal model addressed in Part I, which permits to highlight the main ideas without spurious mechanical complexities. First, the effects of the excitation on periodic solutions are analyzed, focusing on bifurcations entailing their disappearance and playing the role of Koiter critical thresholds. Then, attractor robustness (i.e., large magnitude of the safe basin) is shown to be necessary but not sufficient to have global safety under dynamic excitation. In fact, the excitation strongly modifies the topology of the safe basins, and a dynamical integrity perspective accounting for the magnitude of the solely compact part of the safe basin must be considered. By means of extensive numerical simulations, robustness/erosion profiles of dynamic solutions/basins for varying axial load and dynamic amplitude are built, respectively. These curves permit to appreciate the practical reduction of system load carrying capacity and, upon choosing the value of residual integrity admissible for engineering design, the Thompson practical stability. Dwelling on the effects of the interaction between axial load and lateral dynamic excitation, this paper supports and, indeed, extends the conclusions of the companion one, highlighting the fundamental role played by global dynamics as regards a reliable estimation of the actual load carrying capacity of mechanical systems.
\end{abstract}

Keywords: Load carrying capacity, dynamic excitations, global safety, attractor-basin portraits, dynamical integrity, Koiter, Thompson.

\section{INTRODUCTION}

In the companion paper [1] we put the main contributions to the determination of the practical load carrying capacity of structures into an historical perspective, with the aim of showing how it is reduced by the loss of robustness of stable equilibria. In summary, we have recalled the works of Euler [2], who discovered branching (pitchfork and transcritical) bifurcations in elastic systems (talking in modern language, of course); of Koiter [3], who discovered the structural instability of those bifurcations, i.e. how model imperfections can lower the critical load or, equivalently, how pitchfork and transcritical (branching) bifurcations become saddle-node (snap) bifurcations; and, finally, of Thompson [4-6], who realized that classical stability, based on infinitesimal perturbations of initial conditions, is not enough for practical purposes. 
We have mainly focused on Thompson's contribution, which is the last one and which, in the authors' opinion, is the decisive step toward the final understanding of the problem. Implicitly referring to a global safety approach, Thompson had a double intuition:

(i) If the basin of attraction of a given attractor is not 'large' enough there will be no hope to observe it in real world applications. This can be reformulated by saying that classical local stability refers to infinitesimal changes in initial conditions, while global safety refers to finite changes in initial conditions. Thus, local stability must be complemented by robustness towards variations of initial conditions. In other words, safe basins must have a large enough magnitude.

(ii) Basins of attraction must be topologically 'uncorrupted,' or dynamically integer, i.e., nonfractal. More precisely, for a reliable estimation of the load carrying capacity we have to thrust only on the compact part of the safe basin, ruling out fractality, squeezing and other topological effects which reduce safety without appreciably affecting the magnitude of the safe basin.

Of course, the overall transition from a local stability perspective to a global safety concept has also major implications as regards the involved kinds of global bifurcational events playing a meaningful role in engineering systems, on which possibly interventing - in addition - via a control procedure [7].

Before going on, it is useful to remark that with 'attractor robustness' we basically refer to the safe basin magnitude, while with 'dynamical integrity' we refer to the magnitude of the compact, uncorrupted, part of the safe basin.

The first Thompson point can be addressed, and the main points highlighted, even in the absence of dynamic excitation. This has been done in Part I [1], where, after briefly dwelling on the Euler and Koiter theories, we focused on the reduction of load carrying capacity due to the loss of attractor robustness (Thompson approach). Note that, even if there is no external force, this analysis requires a truly dynamical approach, since it needs analyzing the effect of finite changes of initial conditions, or, equivalently, studying the evolution of safe basins for a growing governing parameters (e.g., the axial load of a beam).

The main ideas have been illustrated in Part I with reference to an archetypal system (see Fig. 1 of [1]), which is of course a crude approximation of real cases, but permits to highlight in a simple way the very basic underlying idea and the main mechanical issues. The simplicity of the model, and the absence of external excitation, have allowed us, for example, to address the matter without solving the equation of motion; not even the time history of the homoclinic orbit, which can be computed in principle (see Sect. 5 forward), has been used.

The aim of this paper is to complement, and complete, Part I [1] by addressing the second Thompson point, which entails considering dynamic excitations. When dynamic excitations are added, in fact, the whole response picture is quite different, more complicate indeed, and, for example, numerical simulations are necessary. Accordingly, the global safety issue becomes more 
involved, from both a theoretical and a practical point of view. The investigation of this issue constitutes the object of this paper, where we assume the lateral force $q$ (the 'imperfection' in our archetypal model) to be of the form

$$
q+q_{1} \sin (\omega t)
$$

i.e., with an harmonic excitation of given frequency $\omega$ added to the static force. This is of course a simplification of the real excitations, but it is again archetypal and sufficient for our purposes. Furthermore, to simulate realistic situations we also consider a damping term acting on the structure, so that the equation of motion becomes (compare with eq. (3) of [1])

$$
\ddot{\beta}+c \dot{\beta}-p \sin (\beta)+\left[1-\frac{1}{\sqrt{1+\alpha \sin (\beta)}}-\left(q+q_{1} \sin (\omega t)\right)\right] \cos (\beta)=0 .
$$

The introduction of a dynamical excitation (and damping) has major effects:

1) the equilibrium position becomes a periodic orbit (as far as the excitation amplitude is sufficiently small, otherwise more complex behaviours may occur);

2) the topology of the safe basin, which becomes basin of attraction, is modified, with the overall picture of variable, local and global, dynamic response becoming very involved and requiring detailed considerations;

3) the issue of dynamical integrity plays now a substantial role in determining the actual load carrying capacity of the system.

These items will be analyzed separately in the following. More precisely, in Sect. 2, still keeping a Koiter point of view, we analyze the effects of the excitation on periodic solutions, focusing on bifurcations entailing their disappearance and thus playing the role of a Koiter critical threshold. No reference is done to basins of attraction. From the one side, this section is propaedeutic to the subsequent analysis, and from the other side, it confirms that the Thompson improvement to the Euler and Koiter ideas holds also, and especially, indeed, in the case of dynamically excited structures.

The basins of attraction are instead considered in the remaining part of the paper. In Sect. 3 the main issues of dynamical integrity, including various adequate measures for evaluating it, are addressed, while in Sect. 4 the reduction of global safety - in terms of attractor robustness and/or basin erosion - is investigated by means of the so-called integrity profiles, an useful graphical tool which permits a visual appraisal of the reduction of load carrying capacity. The effects of both the axial load (Sect. 4.1) and the amplitude of the dynamical excitation (Sect. 4.2) are considered.

The reduction of integrity is triggered by the global bifurcation of the homoclinic orbit surrounding the potential well, which allows penetration of eroding out-of-well fractal tongues into the safe basin of the in-well attractors. Thus, in Sect. 5 the homoclinic bifurcation of the hilltop 
saddle is determined by means of the Melnikov method, which permits an analytical prediction of the threshold where the erosion starts.

The paper ends with some conclusions (Sect. 6) of the present Part II and of the whole work.

\section{PERIODIC SOLUTION SCENARIO: THE KOITER LOAD CARRYING CAPACITY}

According to the 'Koiter philosophy,' which does not consider the basins of attraction in the definition of the load carrying capacity, we address in this section only periodic solutions and their range of existence and stability. We aim at highlighting how the stability threshold of the axially loaded system is affected by the transition from classical equilibria to periodic solutions, entailed by the dynamical excitation.

Before proceeding in the detailed analysis of the periodic orbits, it is necessary to determine the resonant behaviour of the system. The undamped natural circular frequency $\Omega$ (simply frequency in the following) around the stable centre close to the rest position (see Fig. 5 of Part I) can be determined by assuming $\beta(t)=\beta_{e}+\varepsilon \sin (\Omega t), c=0$ and $q_{1}=0$ in (2). Expanding in $\varepsilon$-series up to the first order in $\varepsilon$ we find that the equilibrium point $\beta_{e}$ satisfies eq. (6) of Part I, while $\Omega$ is given by

$$
\Omega^{2}=\frac{-p}{\cos \left(\beta_{e}\right)}+\frac{\alpha}{2} \cos \left(\beta_{e}\right)^{2}\left[1-q-p \tan \left(\beta_{e}\right)\right]^{3} .
$$

Note that for $q=0$ (perfect case) we have $\beta_{e}=0$ and the simple expression

$$
\Omega^{2}=\frac{\alpha}{2}-p
$$

For $\alpha=0.8$ and $q=0.01$, on the other hand, the natural frequency is reported in Fig. 1 as a function of the axial load $p$. We have $\Omega(p=0)=0.623$ and clearly the frequency goes to zero in correspondence of $p=0.302$, i.e. of the Koiter critical load (see Sect. 4 of Part I).

From Fig. 1 we can clearly distinguish three different regions for the forcing frequency $\omega$ :

1) $\omega>0.623$, where for every value of $p$ there will be no resonance;

2) $0.623>\omega>0.3$ (this number is indicative, see Fig. 1), where the resonance occurs for axial loads sufficiently far away from the Koiter threshold;

3) $0.3>\omega$, where the resonance occurs for axial loads around the static buckling load.

For each region, the periodic solutions scenario obtained by a path following algorithm starting from $p=0$ is reported in the following Figs. $2(\omega=0.8), 6(\omega=0.5)$ and $7(\omega=0.2)$. 


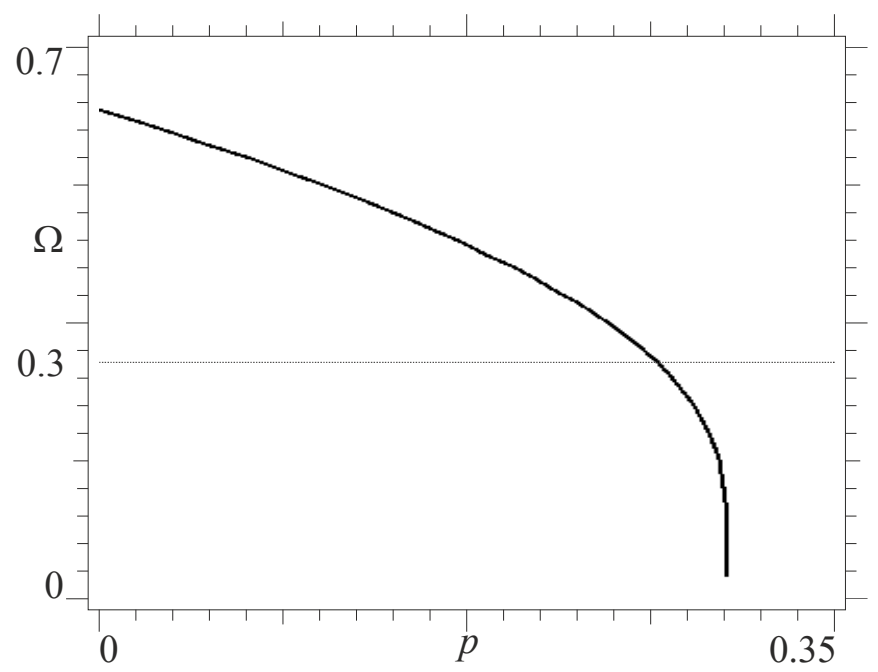

Fig. 1. The natural frequency $\Omega$ as a function of the axial load $p$ for $q=0.01$ and $\alpha=0.8$.

\subsection{Out of the interval of system resonance frequency}

Let us first consider a value of excitation frequency $(\omega=0.8)$ out of the resonance range.

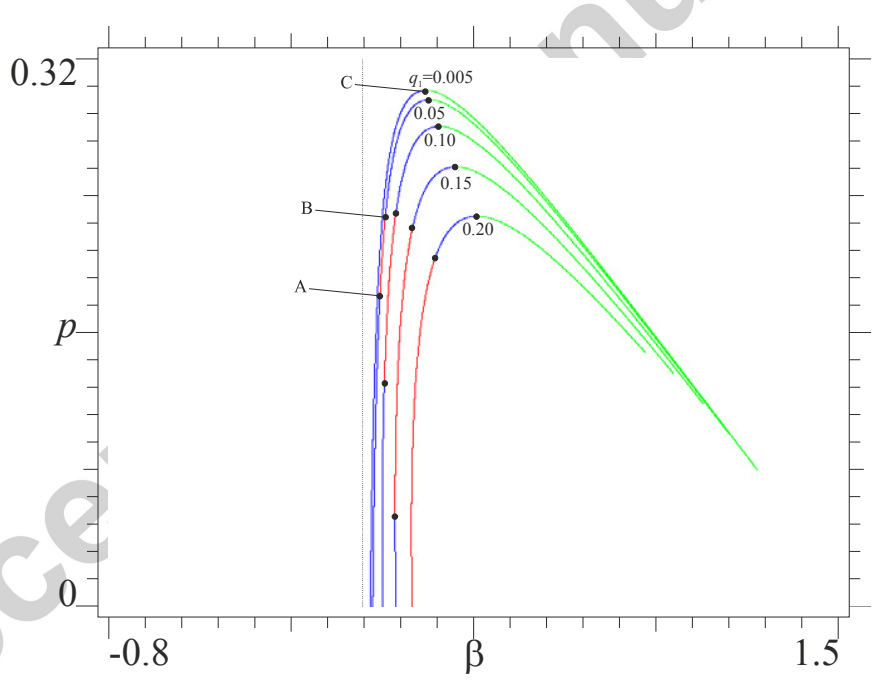

Fig. 2. (color online) Path following of periodic solutions for $c=0.01, q=0.01, \alpha=0.8$ and $\omega=0.8$. Blue=attractor, green/red=regular/flip saddles.

Figure 2 confirms that for increasing axial load there is no resonance. For a very small excitation amplitude $\left(q_{1}=0.005\right)$ the path is similar (and practically can be superimposed) to the static one reported in Fig. 5 of Part I. For increasing $q_{1}$ the paths maintain their general shape, but a period doubling (PD) bifurcation appears (point A, at about $p=0.1813$ for $q_{1}=0.05$; one eigenvalue exits from the unitary circle through -1 ) determining a temporary loss of stability of the main path, which then regains stability by an inverse PD bifurcation (point B; the eigenvalue re-enters the unit circle through -1 ) before undergoing the final saddle node (SN) bifurcation (point $\mathrm{C}$ ). 
The unstable (red) interval becomes larger when increasing $q_{1}$, and eventually it reaches $p=0$, meaning that in the absence of axial load the system is unstable, due to dynamic excitation, and thus unable to carry any axial load. However, this latter case occurs for very large values of $q_{1}$ ( $q_{1}=0.176$, see the forthcoming Fig. 5), which can no longer be considered as irremovable 'imperfections' and thus are out of the scope of the present work.

The brute force bifurcation diagram of Fig. 3 shows that in correspondence of the PD bifurcation the solution jumps to the stable solution in the lateral potential well, around $\beta=\pi$ (see Fig. 4 of Part I), and that, by further increasing $p$, it no longer jumps back to the upper stable part of the main path B-C. This global event, undetectable - though possibly conjecturable - via the sole local stability analysis of Fig. 2, highlights how the PD bifurcation value represents the ultimate load carrying capacity of the system, which is thus strongly reduced in the presence of dynamical excitations for this frequency value.

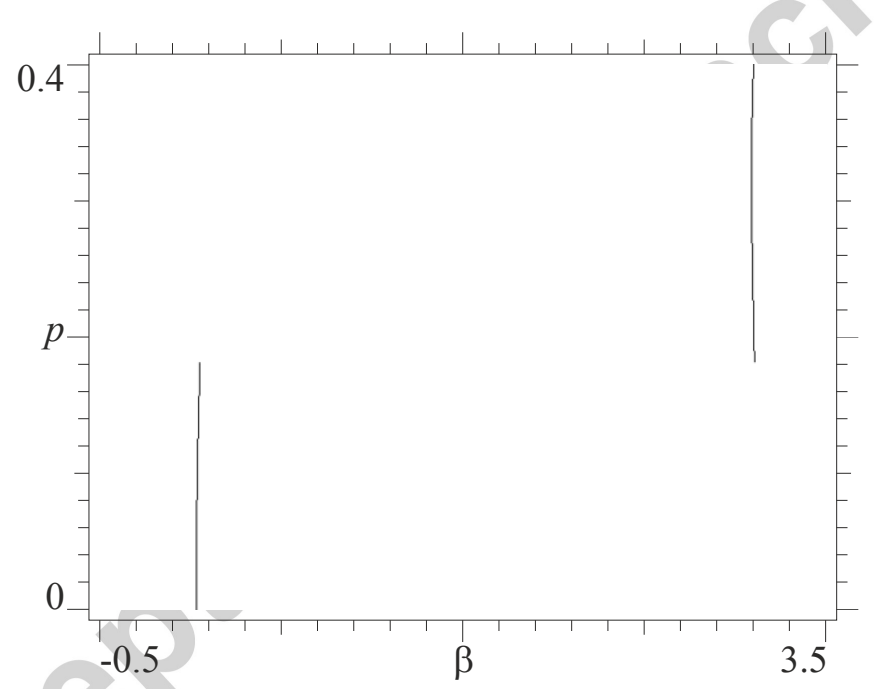

Fig. 3. Brute force bifurcation diagram (for increasing $p$ ) for $c=0.01, q=0.01, q_{1}=0.05, \alpha=0.8$ and $\omega=0.8$.

There are two important consequences of the previous observation. The first is that in the case of (even small) dynamical excitations the load carrying capacity of the system (even in the 'Koiter sense') is strongly reduced, so that considering only static imperfections can be very unsafe. The second is that the load carrying capacity is still determined by local bifurcations. This is a peculiarity of the 'Koiter approach,' which is intrinsically confirmed by the fact that a large use of perturbation methods has been made in the past within the Koiter scientific environment. On the contrary, the 'Thompson approach' involves a global analysis.

We now want to provide an analytical approximation of the equilibrium paths and of the related bifurcations reported in Fig. 2. The basic observation is that the PDs and the SN bifurcations occur for low values of $\beta$, so that we can consider the following approximate governing equation 


$$
\ddot{\beta}+c \dot{\beta}-p \sin (\beta)-\left(q+q_{1} \sin (\omega t)\right)+\left(\frac{\alpha}{2}-p\right) \beta+\left(-\frac{3 \alpha^{2}}{8}+\frac{q+q_{1} \sin (\omega t)}{2}\right) \beta^{2}=0
$$

obtained by expanding the governing equation (2) up to the second order in $\beta$.

We use the harmonic balance method, so that the solution is approximated by the expression

$$
\beta(t)=b_{0}+b_{1} \sin \left(\frac{\omega t}{2}\right)+b_{2} \cos \left(\frac{\omega t}{2}\right)+b_{3} \sin (\omega t)+b_{4} \cos (\omega t)
$$

The $b_{1}$ and $b_{2}$ terms are inserted in (6) to describe the PD bifurcations; indeed, these bifurcations entail a doubling of the period, which is captured by harmonic terms of pulsation $\omega / 2$.

By inserting (6) in (5) we obtain

$$
e_{0}+e_{1} \sin \left(\frac{\omega t}{2}\right)+e_{2} \cos \left(\frac{\omega t}{2}\right)+e_{3} \sin (\omega t)+e_{4} \cos (\omega t)+\ldots=0
$$

According to the harmonic balance method, we equate to zero $e_{0}, e_{1}, e_{2}, e_{3}$ and $e_{4}$, thus obtaining a nonlinear algebraic system of five equations in the five unknowns $b_{0}, b_{1}, b_{2}, b_{3}$ and $b_{4}$.

Equations $e_{1}=0$ and $e_{2}=0$ are, respectively,

$$
\begin{aligned}
& \left(\frac{q_{1} b_{3}}{2}-\frac{\omega^{2}}{4}+\varepsilon_{E}+k_{1}\left(\frac{b_{4}}{2}-b_{0}\right)\right) b_{1}+\left(\frac{q_{1} b_{0}}{2}-k_{1} \frac{b_{3}}{2}-\frac{c \omega}{2}\right) b_{2}=0 \\
& \left(\frac{q_{1} b_{0}}{2}-k_{1} \frac{b_{3}}{2}+\frac{c \omega}{2}\right) b_{1}+\left(\frac{q_{1} b_{3}}{2}-\frac{\omega^{2}}{4}+\varepsilon_{E}-k_{1}\left(\frac{b_{4}}{2}+b_{0}\right)\right) b_{2}=0
\end{aligned}
$$

where

$$
\varepsilon_{E}=\frac{\alpha}{2}-p, \quad k_{1}=\frac{3 \alpha^{2}}{4}-q
$$

Equations (8) constitute a linear homogeneous system in $b_{1}$ and $b_{2}$ having only the solution $b_{1}=b_{2}=0$, apart when its determinant $\operatorname{det}$ (which contains the other unknowns $b_{0}, b_{3}$ and $b_{4}$ ) vanishes. $d e t=0$ is the condition for branching of a period doubled solution from the main path, i.e., the condition for PD bifurcations. Therefore, in the following we assume $b_{1}=b_{2}=0$ to solve the remaining algebraic equations and then monitor det. When it vanishes, we have a PD bifurcation on the main path.

Inserting $b_{1}=b_{2}=0$ in the equations $e_{0}=0, e_{3}=0$ and $e_{4}=0$ we get, respectively,

$$
\begin{gathered}
2 b_{0}^{2}+b_{3}^{2}+b_{4}^{2}-2 \hat{q}_{1} b_{0} b_{3}-4 \hat{\varepsilon}_{E} b_{0}+4 \hat{q}=0 \\
\left(4 b_{0}^{2}+3 b_{3}^{2}+b_{4}^{2}-8\right) \hat{q}_{1}-8 b_{0} b_{3}+8\left(\hat{\varepsilon}_{E}-\delta\right) b_{3}-8 \hat{c} b_{4}=0 \\
\hat{q}_{1} b_{3} b_{4}-4 b_{0} b_{4}+4 \hat{c} b_{3}+4\left(\hat{\varepsilon}_{E}-\delta\right) b_{4}=0
\end{gathered}
$$


which is a system of algebraic quadratic (due to the approximation (5)) equations. In (10) we have defined

$$
\hat{q}_{1}=\frac{q_{1}}{k_{1}}, \hat{q}=\frac{q}{k_{1}}, \hat{c}=\frac{c \omega}{k_{1}}, \delta=\frac{\omega^{2}}{k_{1}}, \hat{\varepsilon}_{E}=\frac{\varepsilon_{E}}{k_{1}}
$$

to simplify the expressions.

The system (10) can be easily solved numerically (indeed, it can even be solved analytically by an algebraic manipulator, but the expressions are so complex to be ineffective in practice). As an example, we report in Fig. 4 the solution for $q=0.01, q_{1}=0.05, \omega=0.8, \alpha=0.8, c=0.01$ and for $p \in[0$, $\left.p_{c r i t}\right]$, which should be compared with the second (from the top) curve of Fig. 2.

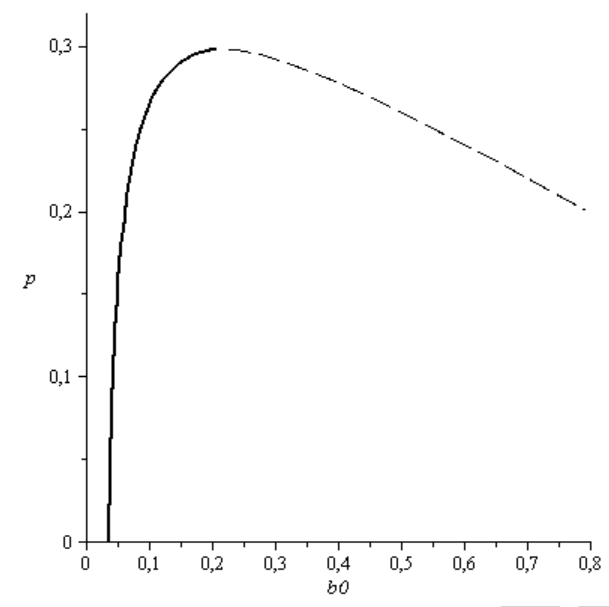

(a)

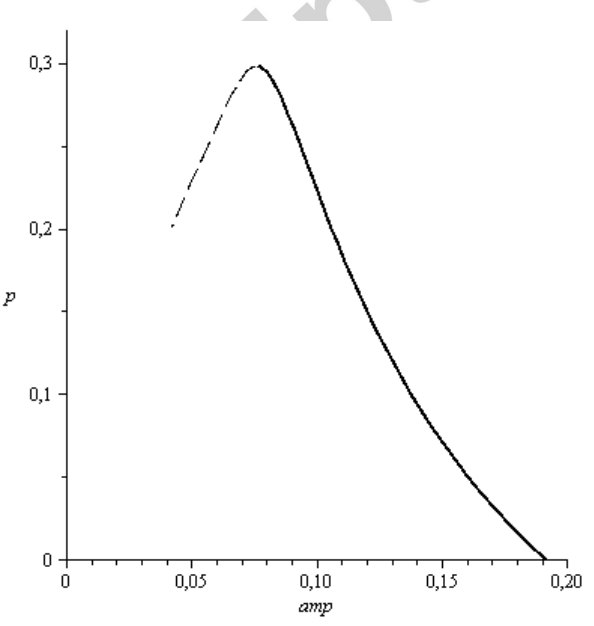

(b)

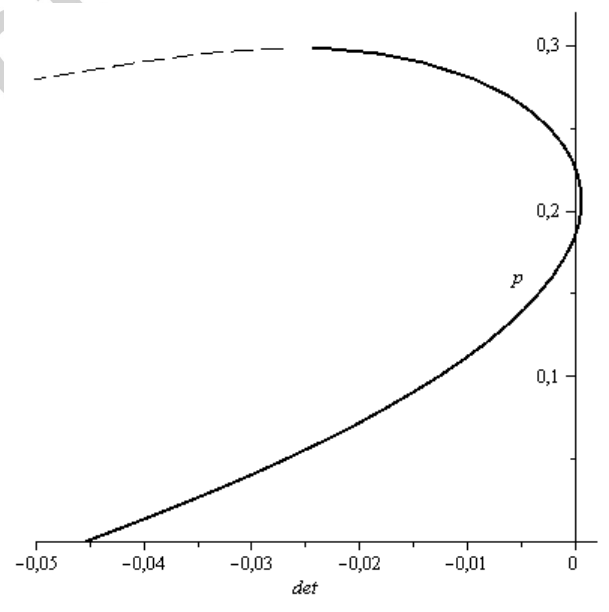

(c)

Fig. 4. (a) $b_{0}$, (b) amp and (c) det as functions of $p$ for $q=0.01, q_{1}=0.05, \omega=0.8, \alpha=0.8$ and $c=0.01$.

In Fig. $4 \mathrm{a}$ it is depicted $b_{0}$, i.e. the average point of the oscillations. Note that due to the quadratic nonlinearities, for $p=0$ it does not coincide with the unexcited equilibrium position $\beta_{e}$ (although it is 
numerically not so different; in fact, they coincide up to the first order in $q_{1}$, see below). It has the same qualitative behavior as the corresponding curve of Fig. 2; however, the comparison cannot be also quantitative, because in Fig. 2 it is reported the point in the stroboscopic Poincarè map, while in Fig. 4a the middle point of the oscillations.

The upper point of the curve of Fig. 4a corresponds to the SN bifurcation. From Fig. 4a we obtain $p_{\text {crit }}=0.2978$, which, when compared with $p_{\text {crit }} \cong 0.296$ obtained from the numerical simulations of Fig. 2, show the effectiveness of the theoretical prediction. Remember that in the static case $p_{K}=0.3014$ (see Sect. 4 of Part I); this means that in this case the dynamic excitation entails only a slight decrease of the SN bifurcation.

In Fig. $4 \mathrm{~b}$ it is reported the amplitude

$$
a m p=\sqrt{b_{3}^{2}+b_{4}^{2}}
$$

of the harmonic oscillations. It is worth to note that it decreases by increasing $p$, and reaches the value $a m p \cong 0.0798$ when $p \rightarrow p_{\text {crit }}$. This behavior has been confirmed by numerical simulations of the governing equation (2).

Finally, in Fig. 4c it is depicted the determinant of system (8). It has clearly two zeros, for $p=0.184$ and $p=0.227$, in perfect agreement with the points $\mathrm{A}$ and $\mathrm{B}$ of Fig. 2 , which are at $p \cong 0.1823$ and $p \cong 0.2282$, respectively.

By drawing several curves like that of Fig. 4c, and by determining on them the zero points of det, it is possible to obtain the loci of PD bifurcations in the $\left(q_{1}, p\right)$ parameters space, which are reported in Fig. 5 together with the upper SN bifurcation. Note that the PD bifurcations exist for $q_{1}>0.007$.

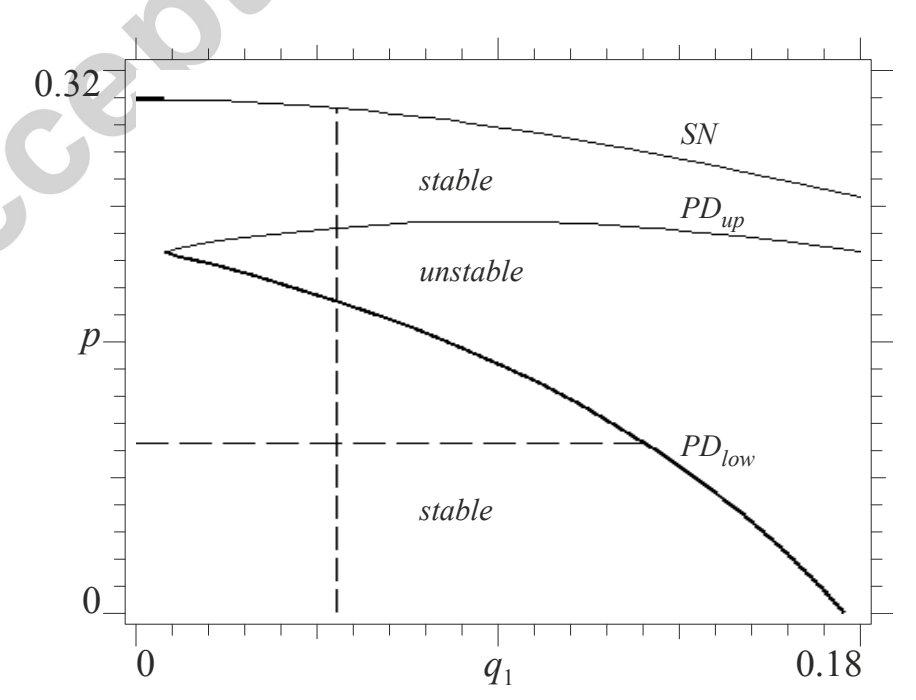

Fig. 5. The curves of SN, upper and lower PD bifurcations in the $\left(q_{1}, p\right)$ parameter plane. $q=0.01, \omega=0.8, \alpha=0.8$ and $c=0.01$. The dashed lines correspond to $p$ and $q_{1}$ values along which the integrity profiles of the forthcoming Figs. 10 and 12 are built. Note that the thick line corresponds to the (discontinuous) Koiter threshold in the presence of dynamic excitation. 
The zone below the lower (thicker) curve $\mathrm{PD}_{\text {low }}$ is the region of stability of the period 1 solution, which can be considered as the region of 'Koiter' dynamic admissibility. This identification could appear rough and too conservative at a first glance, because above $\mathrm{PD}_{\text {low }}$ periodic solutions do exist. In particular, for low values of $q_{1}$, above $\mathrm{PD}_{\text {low }}$ there are period 2 attractors (see the forthcoming Fig. 9e), while above $\mathrm{PD}_{\text {up }}$ the main period 1 attractor regains stability (see Fig. 2). However, the period 2 solution cannot always be considered as a safe (e.g., acceptable) solution, while the upper period 1 solution is not always reachable by increasing $p$. This means that $\mathrm{PD}_{\text {low }}$ is really the Koiter critical threshold in the presence of dynamic excitations.

The interaction between the static $(p)$ and dynamic $\left(q_{1}\right)$ causes of loss of load carrying capacity can be summarized as follows (Fig. 5). For $q_{1}<0.007$ the dynamic excitation has practically no effect: the system fails at about $p_{K}$ (i.e. at about the SN obtained in Part I), since in this range the dynamic excitation lowers this threshold only slightly. At $q_{1}=0.007$ there is a sudden fall down of the critical load, which then continues to decrease smoothly for increasing values of $q_{1}$.

It is also worth noting that, if the static (Koiter) imperfection $q$ is also varied, the overall pattern of the threshold curves remains substantially unchanged from the qualitative viewpoint, although the regions of stable periodic solutions become lower and lower.

\subsection{Within the interval of system resonance frequency}

To illustrate the 'middle' region of excitation frequency, where resonance occurs sufficiently far away from the upper SN, we consider $\omega=0.5$ and report in Fig. 6 the periodic solutions scenario.

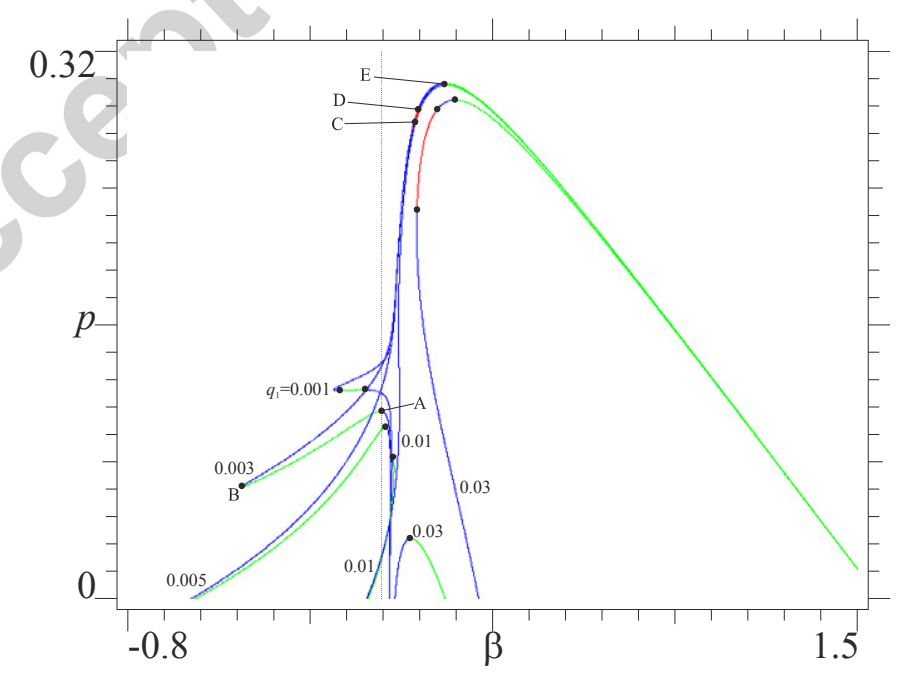

Fig. 6. (color online) Path following of periodic solutions for $c=0.01, q=0.01, \alpha=0.8$ and $\omega=0.5$. Blue $=$ attractor, green/red=regular/flip saddles. 
From (3) we have $\Omega=0.5$ for $p=0.131$, and in fact for that value of $p$ the classical nonlinear resonance behavior is observed in Fig. 6, with two SN bifurcations determining the loss of stability of the non-resonant solution (point A in Fig. 6) and the appearance of the resonant, large amplitude, solution (point $\mathrm{B}$ ). The bending of the resonance curve is toward lower values of $p$, i.e. toward higher values of $\Omega$ (see Fig. 1). For increasing values of $q_{1}$, the bending of the curve becomes higher and higher, along with the peak amplitude value. However, for 'large' values of $q_{1}$ (for example for $q_{1}=0.03$ in Fig. 6) the path is very complicated, and strongly enters the region $p<0$.

In the upper part of the path, a double PD bifurcation is observed (points $\mathrm{C}$ and D) before the final SN (point E). Similarly to what happens in Fig. 2, they appear for a small value of $q_{1}$ (for example they are not present in the path corresponding to $q_{1}=0.001$ ), and then 'propagate' along the path for increasing $q_{1}$.

Determining the load carrying capacity in the present case (still in the 'Koiter philosophy') is quite difficult. In fact, for small values of $q_{1}$ the resonance is not so developed, so that a jump from the non-resonant to resonant periodic attractor (occurring at point $\mathrm{A}$ ) may not be actually dangerous. In this case the load carrying capacity is determined by the upper SN (point E) or, when present, by the lower PD (point C).

For increasing values of $q_{1}$, instead, and even remaining in the range of 'small' values (so that $q_{1}$ can still be considered as an irremovable imperfection), the jump at A may be unsafe, namely it can lead to a resonant solution too far away from the reference one, thus possibly representing the actual load carrying capacity of the system. This is likely to be the case of the 'large' $q_{1}=0.03$ value, for which, in addition, the SN bifurcation value on the non-resonant path is so small that practically the system loses completely its load carrying capacity. Possibly, this excitation amplitude is too large for being considered of interest in practical applications, yet it is worth that this conclusion can be made based on the sole local analysis. Nevertheless, in all of these cases, a global analysis is evidently necessary to fully account for the effects of the dynamic excitation, so that we need to move from the 'Koiter' to the 'Thompson' approach.

Also for the frequency value $(\omega=0.5)$ herein considered an analytical solution by the harmonic balance, or similar methods, is possible. It however requires more care to describe the nonlinear resonances. We do not pursue this point to limit the length of the paper.

The most complicated situation is observed in the third region of excitation frequencies, where the resonance is close to the upper SN. A representative case is reported in Fig. 7 for $\omega=0.2$. We have $\Omega=0.2$ for $p=0.294$ (see Fig. 1), i.e. the resonance is really very close to the upper SN. 


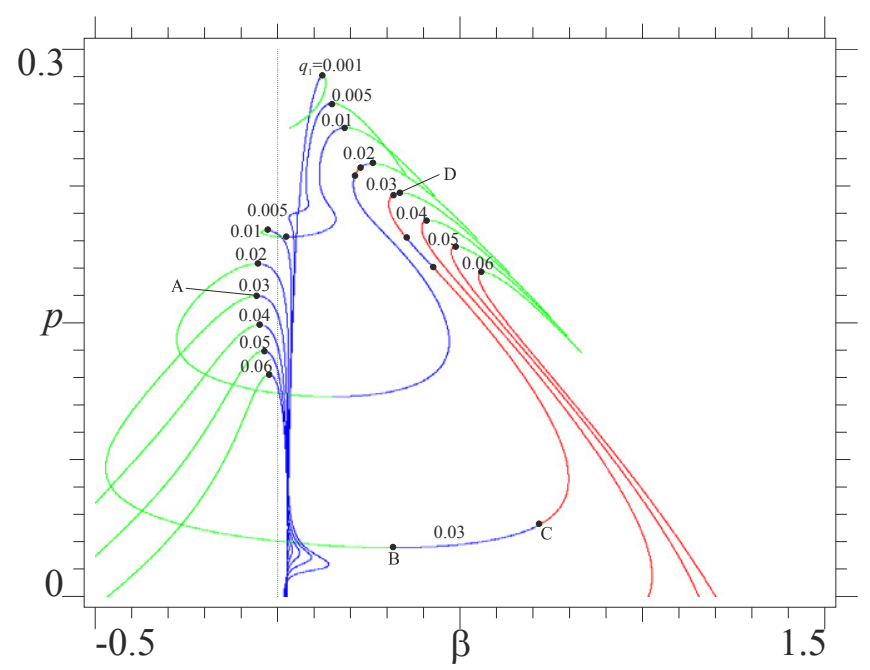

Fig. 7. (color online) Path following of periodic solutions for $c=0.01, q=0.01, \alpha=0.8$ and $\omega=0.2$. Blue=attractor, green/red=regular/flip saddles.

For increasing values of $p$, at about $p \cong 0.0264$ we observe a resonance, which becomes visible and relatively important only for 'large' values of $q_{1}$. By noticing that from (3) we have $\Omega=0.6$ for $p=0.0269$ we see that this is an order-3 superharmonic resonance. It is not so pronounced because the quadratic nonlinearities are dominant in this system.

When $q_{1}$ is very small (e.g. $q_{1}=0.001$ ), by increasing $p$ we directly reach the upper SN determining the load carrying capacity of the system in this case. For larger (but still small) values of $q_{1}$ we note a resonant behavior. For $q_{1}=0.005$ this occurs at $p \cong 0.209$. Again we appeal to (3) to note that $\Omega=0.4$ for $p=0.212$, so that this is an order- 2 superharmonic resonance. Contrary to the previous order-3 resonance, and due to the quadratic-like behavior of the nonlinearity, it is now very pronounced, and its nonlinear effects become visible for increasing values of $q_{1}$; for example, for $q_{1}=0.01$ the two SN bifurcations determining the nonlinear hysteresis loop are still present (they are marked with points in Fig. 7).

For larger values of $q_{1}$ the nonlinear behavior becomes more and more important, and the bending of the resonance curves becomes predominant, and quite complicated, indeed. For example, in the case $q_{1}=0.03$ (the last path completely within the window of Fig. 7), after the SN A where the small amplitude oscillation loses stability there is a long unstable path leading to the SN $\mathrm{B}$ where a large amplitude periodic orbit is born. Then, there is a small range where the resonant cycle is stable; it loses stability by a PD bifurcation (at C), then there are three other PDs at which alternatively the oscillation regains and loses stability, up to the upper SN D where stability is definitely lost.

In all these cases, due to the occurrence of clearly unsafe jumps, the load carrying capacity is certainly given by the $p$ threshold for the SN A, when present, i.e. for moderately large values of $q_{1}$. It is evidently much lesser than the static Koiter load, and governed by a superharmonic resonance. 
Here, in contrast to the case of Fig. 6 where it is dominant, the main resonance is too close to the upper SN to play a clearly visible role. More precisely, it is not even activated for 'large' values of $q_{1}$, because in this case the path remains quite far apart from the threshold $p=0.294$.

The main overall conclusion of this section is that in the presence of dynamical excitation the response scenario exhibiting periodic orbits becomes much more complex than the static one, even in the case of external frequencies out of the resonance interval. In fact, both secondary (PD) bifurcations and various jump phenomena ensuing from resonances reduce the load carrying capacity even for relatively small excitation amplitudes.

But the situation is even more complex. In fact, as we already saw in Sect. 5 of Part I when considering the system global safety in the absence of dynamical excitations, close to local bifurcations the basins of attractions shrink to zero, so that the practical (Thompson) load carrying capacity is smaller than that discussed in this section. To determine it we need a global approach fully accounting for the actual dynamical integrity of the system. This will be the subject of the next two sections.

\section{ATTRACTOR-BASIN SCENARIO: THE DYNAMICAL INTEGRITY PERSPECTIVE}

In this section we want to reproduce the analysis in Sect. 5 of Part I in the presence of dynamical excitations. From a practical point of view the major novelty is that the safe basins, which are now basins of attraction, can no longer be computed analytically, and need heavy numerical simulations, so that the analysis becomes quite time consuming.

The most important theoretical changes are instead associated with the possible fractalization of basins of attraction, which is illustrated in Fig. 8, where, by comparing Fig. 8a with Fig. 8b, it is clearly seen the fractalization due to dynamic excitation. The coexistence (and competition) of more attractors, which is another important consequence of the dynamic excitation, is also illustrated in Fig. 8b, where five different basins are visible (see the figure caption for their description). From Fig. $8 \mathrm{~b}$ it is apparent how the basins of other attractors (in particular the red one) influence the basin (the white one) of the periodic attractor of interest (which is the perturbation of the rest position) through distributed fractalization. 


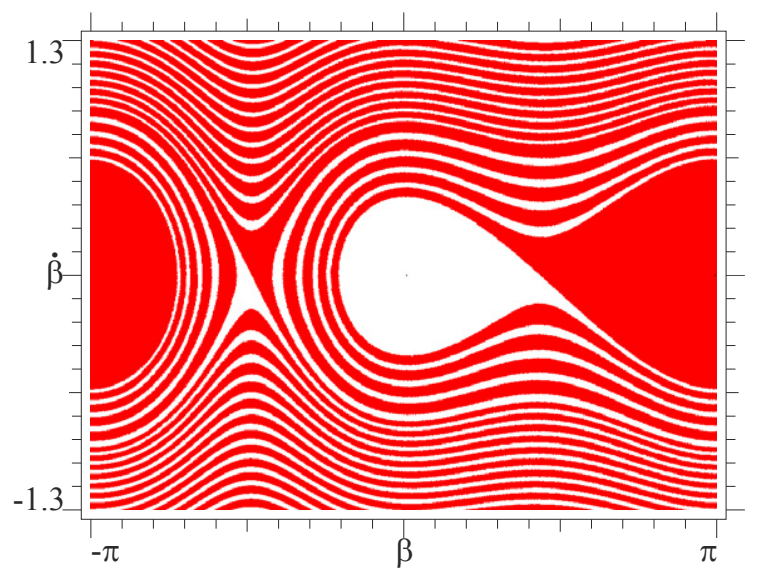

(a)

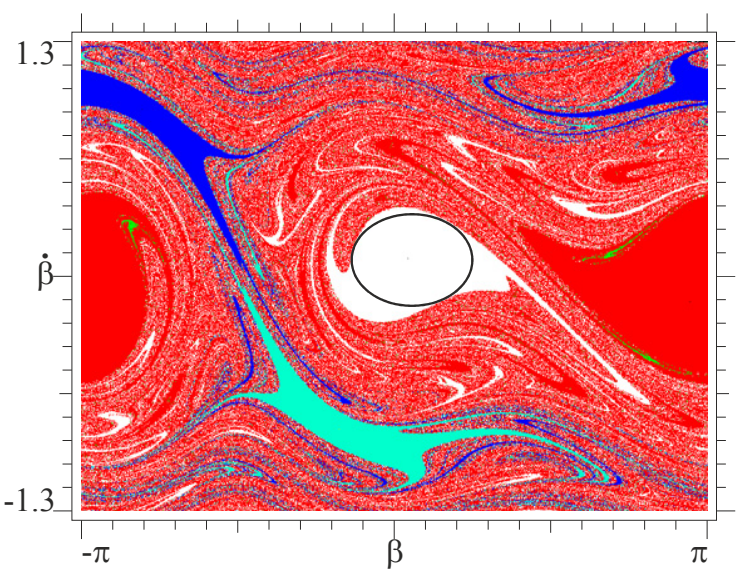

(b)

Fig. 8. (color online) Basins of attraction for $c=0.01, q=0.01, \alpha=0.8, p=0.05$ and $\omega=0.8$. (a) $q_{1}=0$ (without dynamic excitation) and (b) $q_{1}=0.05$ (with dynamic excitation). White $=$ basin of a period 1 oscillation around $\beta=0$ - the attractor of interest; red $=$ basin of a period 1 oscillation around $\beta=\pi$; blue $=$ basin of a clockwise rotation $(\dot{\beta}>0)$; cyan $=$ basin of an anti-clockwise rotation $(\dot{\beta}<0)$; green $=$ basin of a period 6 oscillation around $\beta=\pi$ (it is just after a PD bifurcation of a period 3 oscillation).

As Fig. 8b clearly shows, due to fractality the whole basin of attraction is no longer a safe, i.e. integer, region - as in the unexcited case - where the attractor is robust against dynamical perturbations constituted by changes in the initial conditions. In fact, smaller (with respect to the unexcited case) perturbations are sufficient to lead to another attractor.

To overcome this point, full recourse has to be made to the dynamical integrity concept, by also properly addressing the matter of a reliable choice of how to measure the integrity. The Global Integrity Measure $(G I M)[4,8]$, already referred to in Part I [1] to deal with the robustness of stable equilibria, is the magnitude (the area in 2D) of the basin of attraction normalized with respect to a reference unexcited case. However, it is unable to take the fractality of the basin of attraction into account. The so-called Local Integrity Measure (LIM) [5] is the normalized minimum distance between the attractor and its basin boundaries (normalized means divided by a reference number, usually the unperturbed one, so that LIM is a dimensionless number, like GIM). This is a measure of the compact part of the basin around the attractor, so it succeeds in ruling out the fractal part of the basin from the dynamical integrity considerations. In spite of this valuable property, it also has two main drawbacks. The first is that it is computationally onerous, especially for chaotic attractors, while the second is that it works only for basins of attraction and cannot be generalized to different definitions of safe basins, which in a modern viewpoint can be considered also as subsets of the phase space sharing some common properties, e.g. a confined response, instead of the mere set of initial conditions approaching a common attractor.

The previous disadvantages have been surmounted by the introduction of the so-called Integrity Factor (IF) [7-9], which is the normalized radius of the largest hyper-sphere (circle in 2D) belonging to the safe basin. Like LIM, this measure is able to rule out the fractal part of the basin 
from the dynamical integrity, but contrary to LIM it is computationally easy (it requires just a command in Matlab ${ }^{\mathrm{TM}}$ ) and is valid for any kind of safe basin, not only for basins of attraction. The circle related to the $I F$ is reported in Fig. $8 \mathrm{~b}$ for the attractor of interest.

It is important to remark that in the absence of fractal basins boundaries GIM, LIM and IF are somehow equivalent; only in the presence of fractality the use of LIM or IF may become necessary.

To illustrate qualitatively the reduction of dynamical integrity of the periodic attractors entailed in the axially loaded system by a lateral dynamical excitation, we report in Fig. 9 a succession of basins of attraction for increasing values of $p$ and for fixed values of the other parameters. This picture is the 'equivalent,' in the dynamical case, of Fig. 7 of Part I. The comparison between these two figures highlights the more complex behaviour under dynamic excitation. The periodic attractor in the central part (which is the attractor of interest for being the perturbation of the rest position) in Fig. 9 corresponds to the curve $q_{1}=0.05$ of Fig. 2 and to Fig. 4 .

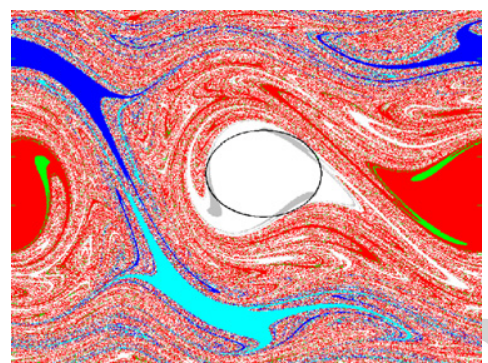

(a)

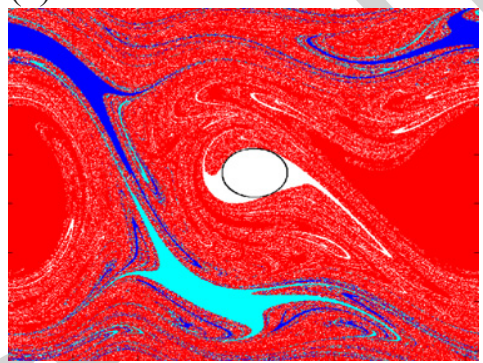

(c)

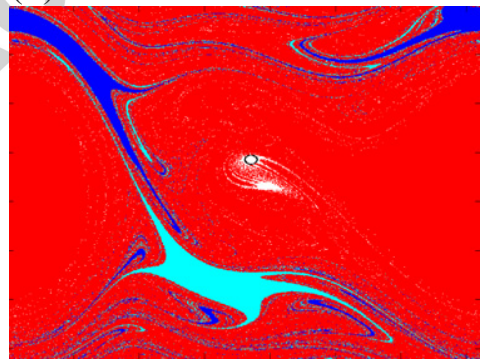

(e)

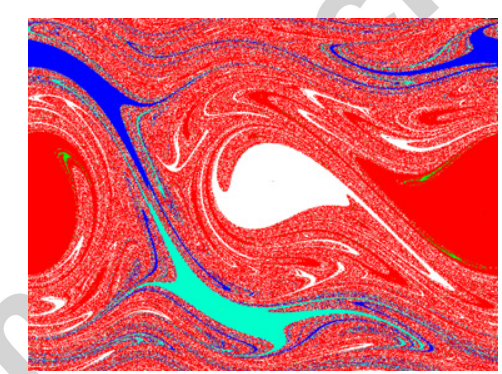

(b)

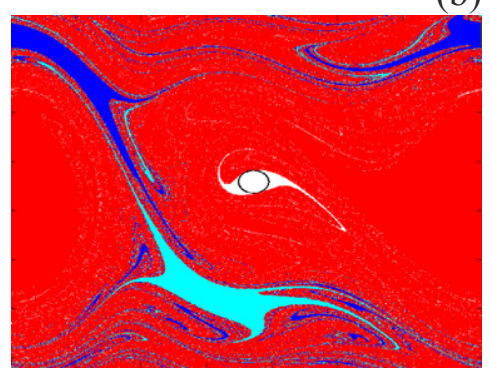

(d)

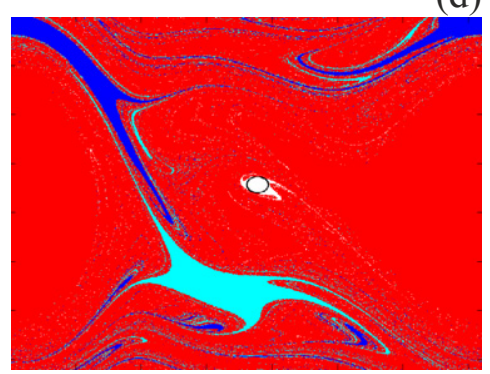

(f)

Fig. 9. (color online) Basins of attraction for $c=0.01, q=0.01, \alpha=0.8, q_{1}=0.05, \omega=0.8$ and for increasing values of $p$. (a) $p=0.00$; (b) $p=0.05$; (c) $p=0.10$; (d) $p=0.15$; (e) $p=0.20$ and (f) $p=0.25$. The window is $-\pi<\beta<\pi$ and $1.3<\dot{\beta}<1.3$ in every picture. 
Apart from the overall reduction of size of the basin of attraction (both the overall size and the size of its compact part, see the circles related to $I F$ ) and the extended fractality, Fig. 9 highlights other interesting points, which are now illustrated.

In Fig. 9a there are two attractors in the central potential well, one of period 1 and one of period 3. Actually, the period 3 attractor soon disappears (it is no longer present in Fig. 9b), but its presence permits in any case to address a theoretical issue. In fact, in this and similar cases we can alternatively consider the basin of attraction of a single attractor, or the union of the basins of all attractors belonging to the potential well. In the first case we are addressing the dynamical integrity of a given attractor, in the latter the dynamical integrity of a potential well. Which one is the more interesting point of view depends on the application at hand, and there are no general rules; however, one must be aware of the difference. As an example, in Fig. 9a we have considered as safe basin the union of the basins of the two attractors belonging to the potential well (as clearly shown by the IF circle).

In Fig. 9e the main period 1 attractor has lost stability by a PD bifurcation (see Figs. 2 and 4c) and, accordingly, a period 2 attractor is observed. This has strong consequences in terms of dynamical integrity. In fact, due to the fractal part separating the two compact parts of the basin (each one around the two points of the attractor in the stroboscopic Poincarè section), the radius of the largest circle, and the associated integrity, suddenly decreases. This fact, which is better detected by $I F$ than by GIM, is one (representative) example of several mechanisms determining the sudden, dangerous, reduction of dynamical integrity.

The qualitative analyses of Fig. 9 must be complemented by a quantitative analysis, which is achieved by the construction of integrity profiles which are actual dynamic counterparts of the robustness profiles of stable equilibria under finite-amplitude imperfections reported in Figs. 8 and 9 of Part I [1]. Here, however, the governing parameters mainly influencing the dynamical integrity are two, the axial load $p$ and the dynamic excitation $q_{1}$ (see Fig. 5), and we have to address the effects of both of them.

\section{INTEGRITY PROFILES AND THE THOMPSON LOAD CARRYING CAPACITY}

Having in mind the reference case $q=0$ and $q_{1}=0$, the curve $\operatorname{GIM}(p)$ is the upper one reported in Fig. 9 of Part I [1]. Since there is no dynamical excitation, and thus no associated fractality, the curve $\operatorname{IF}(p)$ is not needed in this case, and indeed it was not reported therein. In fact, in Part I, the curve $\operatorname{GIM}(p)$ has been said to merely represent a profile of robustness of the stable equilibrium position under finite size perturbations. 


\subsection{Increasing axial load at fixed dynamic excitation: robustness profiles}

The aim of this subsection is to replay the analysis of Part I [1], i.e. to see how the global safety decreases when increasing the axial load, but now considering the effect of the dynamic excitation. In particular, we want to discuss how the dynamic excitation modifies the Thompson critical load.

Both the $\operatorname{GIM}(p)$ and $\operatorname{IF}(p)$ for $q_{1}=0.05$ are reported in Fig. 10, where the area and the radius of the circle are normalized with respect to the values for $p=0$ and $q_{1}=0$. The $G I M$ curve for the unexcited case $q_{1}=0$ is also reported for comparison. Like that profile, the curves for $q_{1} \neq 0$ basically represent profiles of robustness of the reference solution, which is now periodic. A difference is in the role possibly played by the fractal erosion, which is however, herein, a substantially localized phenomenon, as somehow expectable given the actual non-evolutionary, static indeed, character of the present control parameter. The profiles have been built by constructing several basins of attraction for increasing values of $p$, and measuring the area of the basin and the radius of the largest circle. The safe basin is the union of the basins of all attractors belonging to the potential well. Some of the basins used for its construction are reported in Fig. 9, while the periodic attractor scenario is reported in Fig. 2 (numerical) and Fig. 4 (analytical).

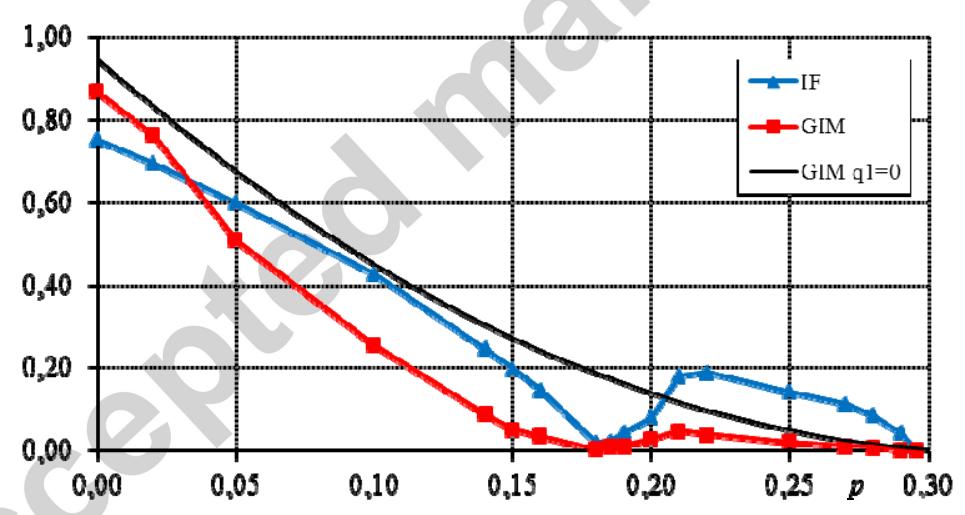

Fig. 10. (color online) The robustness profiles for $I F$ and GIM. $c=0.01, q=0.01, \alpha=0.8, q_{1}=0.05$ and $\omega=0.8$. The GIM curve for the unexcited case $q_{1}=0$ is also reported for comparison.

The first observation which follows from Fig. 10 is that the GIM with dynamic excitation is systematically well below the corresponding GIM curve in the absence of excitation, although the two curves share a similar qualitative behaviour (only $I F$ has a different qualitative behaviour). This shows the meaningful effects of the excitation in reducing the safety of the system. To have a quantitative idea of the decrement, let us suppose that the admissible GIM is $20 \%$, which is quite a small value, indeed. We then obtain a 'dynamic' practical stability (Thompson) threshold $p_{T}\left(q_{1}=0.05\right)=0.116$ which is $66 \%$ of $p_{T}\left(q_{1}=0\right)=0.175$, namely the dynamic excitation almost halves the 'static' practical stability (Thompson) threshold. Note that $p_{T}\left(q_{1}=0.05\right)$ is also $64 \%$ of the 
practical (i.e., 'dynamic') Koiter critical load $p_{K} \cong 0.18$ (corresponding to the $\mathrm{PD}_{\text {low }}$ bifurcation of the path of Fig. 2, see Sect. 2.1) and 39\% of the theoretical (i.e., nearly 'static') Koiter critical load $p_{K} \cong 0.296$ (corresponding to the final SN bifurcation of the path of Fig. 2). This means that, accounting for also the global effects which characterize the actual dynamic response, the practical stability threshold $p_{T}\left(q_{1}=0.05\right)$ is about one third of the theoretical Koiter critical load. Overall, it is apparent that full consideration of the effects of a dynamic excitation entails a strong reduction of the actual critical threshold and of the structural performance.

In Fig. 10, initially $I F<G I M$ because we are around $p=0$ where this happens for every value of $q_{1}$ (see forthcoming Fig. 11). But when $p$ increases above $p \cong 0.035$, GIM passes below $I F$ and then decreases faster than $I F$. The temporary loss of stability of the period 1 attractor (see the path in Fig. 2) is clearly highlighted by a strong reduction of the integrity, which almost vanishes, visible in both curves. In particular, GIM accounts for the progressive - yet smooth - reduction of robustness of the periodic attractor entailed by the decreased magnitude of its basin (see Fig. 9), whereas $I F$ is able to catch the sudden reduction of basin compactness occurring at $p \cong 0.18$ (Figs. 9d and 9e). From a practical point of view this means that indeed, due to global effects, we cannot actually reach the upper stable part of the local bifurcation path by increasing $p$, so that, as previously anticipated, the practical Koiter critical threshold can be identified with the $\mathrm{PD}_{\text {low }}$ threshold.

In terms of load carrying capacity, we can conclude again that the Thompson 'critical threshold' - now computed either referring to GIM or to IF and not only to GIM as in Part I - is lower, and possibly much lower, than the Koiter one. This is especially true for GIM because, as in the case $q_{1}=0$, GIM reaches the Koiter threshold $\mathrm{PD}_{\text {low }}$ by a practically horizontal slope, which entails (and indeed is the root of the) sensitivity to dynamical perturbations.

After the interval between the two PD bifurcations, the main period 1 oscillation recovers stability (Fig. 2) and integrity before definitely disappearing through the final SN bifurcation. This is particularly evident with $I F$ which is again able to catch the recuperated compactness of the basin from about Fig. 9e to Fig. 9f. Yet, this behavior has no effects in terms of load carrying capacity since it practically ends (in the Koiter sense) at $\mathrm{PD}_{\text {low }}$

Figure 10 shows that GIM is the most conservative measure of integrity. The inequality $G I M<I F$ is quite uncommon and has not been systematically observed in other works [7-10], where, however, the driving parameter is usually the dynamic excitation amplitude. A justification is reported in the following Subsection 4.2, based on the different depth of the two potential wells.

\subsection{Increasing dynamic excitation at fixed axial load: erosion profiles}

The curves in Fig. 10 are robustness profiles for increasing axial load $p$, obtained at a fixed nonvanishing value of the excitation amplitude $q_{1}$. They also highlight some (minor) effect associated 
with the topological erosion of the reference attractor basin. However, in terms of dynamical integrity, actual erosion profiles are obtained by increasing the excitation amplitude $q_{1}$, at fixed values of the axial load $p$ [7-10]. In the absence of axial load $(p=0)$ both the $\operatorname{GIM}\left(q_{1}\right)$ and $\operatorname{IF}\left(q_{1}\right)$ are reported in Fig. 11, where again the area and the radius of the circle are normalized with respect to the values for $p=0$ and $q_{1}=0$, and the safe basin is the union of the basins of all attractors belonging to the potential well.

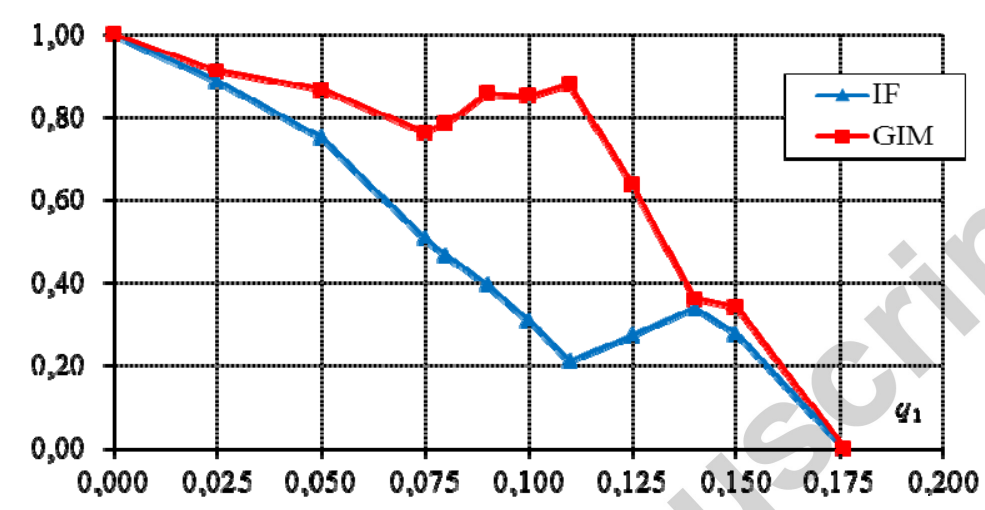

Fig. 11. (color online) The erosion profiles for $I F$ and GIM. $c=0.01, q=0.01, \alpha=0.8, p=0$ and $\omega=0.8$.

Both curves start from 1, due to the normalization, and end where the last attractor disappears at the value of the limiting (i.e., purely dynamic) 'Koiter' load carrying capacity $q_{1, K} \cong 0.177$ (see Fig. 5 at $p=0$ ): this is the escape load of the system. The central part of the two curves shows a quite distinct behaviour. In particular, $I F$ is lower than GIM, sometimes much lower, meaning that there is a large fractal part of the basin which does not contribute to the dynamical integrity. This is an example in which using GIM would lead to unsafe conclusions, and $I F$ is absolutely needed for a reliable integrity evaluation. This behaviour has been observed repeatedly in previous authors' works [7-10], although it is not general, as we have seen in Fig. 10 which, however, is not an erosion profile.

The curves in Fig. 11 confirm once more that the practical (Thompson) load carrying capacity is lower, and possibly much lower, than the Koiter one. This depends of course on the admissible value of residual integrity, and on the employed integrity measure. For example, if we admit that $40 \%$ of the initial integrity is still acceptable, we have $q_{1, T}^{G I M} \cong 0.14$ (corresponding to $79 \%$ of the Koiter threshold) and $q_{1, T}^{I F} \cong 0.08$ (45\% of the Koiter threshold). If, instead, one needs a practically uneroded situation (e.g. $80 \%$ of the initial value), then $q_{1, T}^{G I M} \cong 0.115$ (corresponding to $65 \%$ of the 
Koiter threshold) and $q_{1, T}^{I F} \cong 0.04$ (22\% of the Koiter threshold). These numbers further confirm that, in this case, $I F$ is more conservative than GIM to a large extent.

The GIM curve with $q_{1}=0$ in Fig. 10 and the two (GIM and $I F$ ) curves in Fig. 11 refer respectively to the coordinate axes delimiting the stable region in the $\left(q_{1}, p\right)$ parameters space of Fig. 5, and correspond to the two limit cases of no dynamic excitation $\left(q_{1}=0\right)$ and no axial load $(p=0)$. To have an idea of what happens in other parts of that region, in addition to the other curves in Fig. 10, we report in Fig. 12 the erosion profiles drawn along the horizontal dashed line of Fig. 5. Again we have used the case $p=0$ and $q_{1}=0$ to normalize the curves - which therefore do not start from 1 for $q_{1}=0$ (Fig. 12, where $p=0.1$ ) and for $p=0$ (Fig. 10, where $q_{1}=0.05$ ) - so that they are comparable with each other and with Fig. 11.

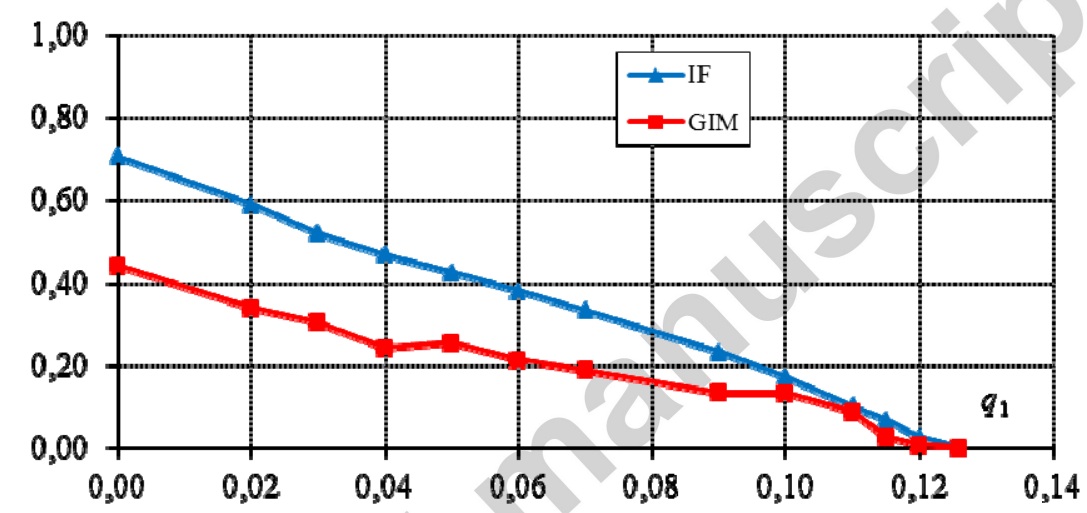

Fig. 12. (color online) The erosion profiles for $I F$ and GIM. $c=0.01, q=0.01, \alpha=0.8, p=0.1$ and $\omega=0.8$.

The main difference between the curves of Figs. 11 and 12, which are both obtained for increasing $q_{1}$ but for different values of $p$, is that now $I F$ is always higher than GIM, and thus the latter is the most conservative measure.

This different behaviour can be explained by noting that for $p=0$ the potential wells around $\beta=0$ and $\beta=\pi$ have the same depth, as shown in Fig. 2a of Part I. Thus, roughly speaking, they have the same 'degree of attractivity,' so that the latter does not capture the basin of the former by an 'unbalanced potential effect,' and the safe basin (as well as its GIM) decreases 'slowly.' In parallel, however, the reciprocal fractalization, triggered by the homoclinic bifurcation of the hilltop saddle proceeds, so that $I F$ strongly reduces. $I F$ is thus lesser than GIM.

For $p=0.1$, on the other hand, the potential well around $\beta=\pi$ is much deeper than that around $\beta=0$ (Fig. 2a of Part I), and thus it is much more 'attractive.' So attractive, evidently, to capture the area of the safe basin from the other well for whatever $q_{1}$ value, thus entailing an overall reduced value of its GIM. An example is reported in Fig. 8 (which however refers to $p=0.05$ ), where the red area already larger than the white area for $q_{1}=0$ (Fig. 8a) - tends to progressively erode it for $q_{1} \neq 0$ (Fig. 
8b). This phenomenon is certainly amplified by the approximation of the numerical simulations, whose outcomes in the fractal basin boundaries are more 'attracted' toward the deeper, more robust, well.

The fractalization of course proceeds also in this case (we are above the hilltop homoclinic bifurcation threshold, see Sect. 5), but evidently it is not so fast to cancel the 'unbalanced potential effect' before getting to the final escape, so that $G I M<I F$ in the whole $q_{1}$ range. This occurs also for higher values of $p$, where the lateral well becomes deeper and deeper (Fig. 2a of Part I), see Fig. 10.

To confirm the previous interpretation, we have built also the robustness profiles of the attractor in the lateral potential well (Fig. 13) and, as expected, we obtain $I F<G I M$. Note that, in this case, both profiles increase with $p$. This highlights the competition occurring between the safe basins of the two coexisting potential wells: the integrity of the central one (around $\beta=0$ ), which is actually eroded, decreases, whereas the integrity of the lateral well (around $\beta=\pi$ ) increases. This shows how, in general, the matter of system dynamic integrity is rather involved and has to be evaluated also with respect to the operating conditions to be considered acceptable for the system at hand, in practical situations.

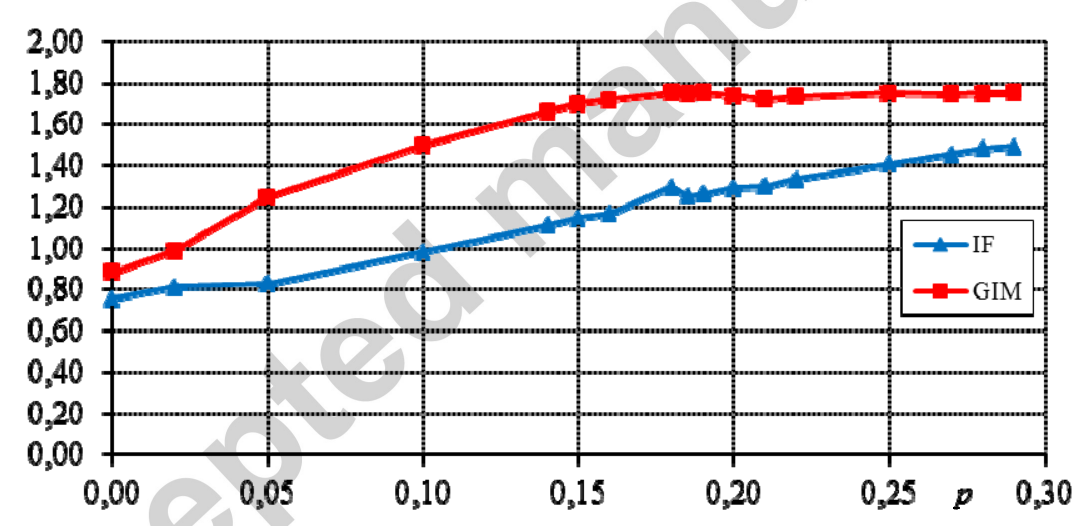

Fig. 13. (color online) The robustness profiles for $I F$ and $G I M$ for the attractor in the lateral well. $c=0.01, q=0.01, \alpha=0.8$, $q_{1}=0.05$ and $\omega=0.8$.

To close this section we summarize in Fig. 14 the integrity profiles obtained so far using GIM, which provide a measure either of the reduction of attractor robustness (for varying $p$ ) or of the extent of basin erosion (for varying $q_{1}$ ). The figure highlights the coupling effects entailed on the system load carrying capacity by the coexistence of axial load and lateral dynamic excitation, and provides a clear view of how the integrity decreases up to vanishing when approaching the limit curve in the $\left(q_{1}, p\right)$ parameters plane, which is just the $\mathrm{PD}_{\text {low }}$ curve of Fig. 5. This limit curve highlights the strong reduction both of the (Koiter) practical critical load owed to the presence of a dynamic excitation $\left(q_{1} \neq 0\right)$ and of the (Thompson) escape dynamic excitation owed to the presence of a static axial load $(p \neq 0)$. 


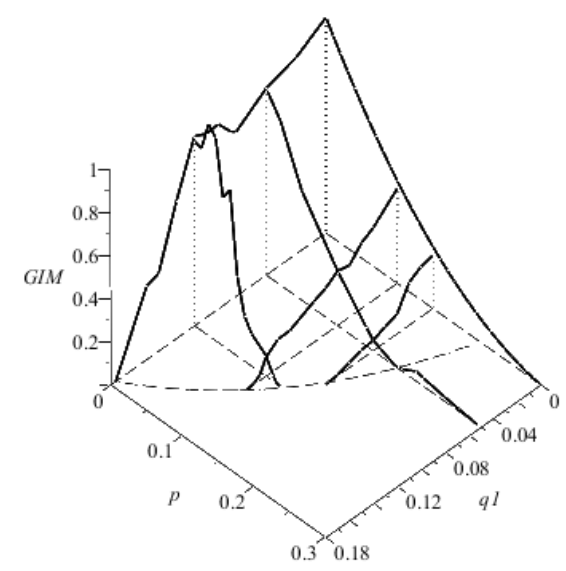

Fig. 14. A tridimensional view of the robustness-integrity profiles. $c=0.01, q=0.01, \alpha=0.8$ and $\omega=0.8$.

Finally, we note that in Sects. 3 and 4 only the case of a non-resonant excitation frequency (see Sect. 2.1) has been analyzed in terms of dynamical integrity. The two cases of resonant frequency discussed in Sect. 2.2 can be analyzed along the same lines, but this is not reported to limit the length of the paper. We only note that the considered case is the simplest one (compare Figs. 2, 6 and 7), yet it exhibits already a quite complex integrity behavior. The other cases will be certainly more involved.

\section{ANALYTICAL PREDICTION OF THE HOMOCLINIC BIFURCATION}

In the previous sections we have seen the major role played by the fractality of the basins of attraction in the dynamical integrity issue, and in determining the associated Thompson load carrying capacity. This phenomenon is triggered by the global bifurcation of the homoclinic loop surrounding the potential well, a topological event which can be studied analytically by the Melnikov method $[11,12]$. To this aim, we recover the treatment of the homoclinic orbit sketched in Part I [1] to accomplish the relevant, limited, goals, and analyze its dependence on the system parameters thus obtaining a suitable theoretical prediction of the homoclinic bifurcation.

Before going on, it is worth to note that the homoclinic bifurcation of certain saddles is the threshold for the onset not only of fractal basins boundaries, as already said, but also of other related complex phenomena such as chaotic saddles, chaotic transients and sensitivity to initial conditions.

To apply the Melnikov method we rewrite (2) in the form ( $c=\varepsilon \hat{c}$ and $\left.q_{1}=\varepsilon \hat{q}_{1}\right)$

$$
\begin{gathered}
\ddot{\beta}+V^{\prime}(\beta)=\varepsilon\left[-\hat{c} \dot{\beta}+\hat{q}_{1} \sin (\omega t) \cos (\beta)\right], \\
V(\beta)=\frac{[1-\sqrt{1+\alpha \sin (\beta)}]^{2}}{\alpha}-p[1-\cos (\beta)]-q \sin (\beta),
\end{gathered}
$$


i.e., as the sum of the conservative system equation plus small perturbations accounting for the damping and the dynamical excitation ( $V$ is the system potential, see Part I [1]). The unperturbed system has an homoclinic orbit $\beta_{h}(t)$ (see Fig. 6a and Fig. 7 of Part I) encircling the stable equilibrium point $\beta_{e}$. This solution is homoclinic to the saddle $\beta_{s}$ just on the right of $\beta_{e}$ (see Fig. 4 of Part I, for the general case of non-zero static imperfection), and it can be obtained by solving

$$
\dot{\beta}_{h}(t)= \pm \sqrt{2\left[V\left(\beta_{s}\right)-V\left(\beta_{h}(t)\right)\right]}
$$

an equation which comes from the energy balance upon considering that $\dot{\beta}=0$ at the saddle. Let $\beta_{o}$ be the first solution of $V\left(\beta_{s}\right)=V(\beta)$ on the left of $\beta_{e}$; it is the minimum value attained by the homoclinic orbit. Without loss of generality we can assume $\beta_{h}(t)=\beta_{h}(-t)$ (symmetry condition) so that $\beta_{h}(0)=\beta_{o}$. Eq. (14) then provides the inverse function $t=t(\beta)$ :

$$
t=\int_{\beta_{o}}^{\beta} \frac{d z}{\sqrt{2\left[V\left(\beta_{s}\right)-V(z)\right]}} .
$$

The Melnikov function, which measures the first order (with respect to $\varepsilon$ ) distance between the perturbed stable and unstable manifolds is given by $[11,12]$

$$
M(m)=\int_{-\infty}^{\infty} \dot{\beta}_{h}(t)\left[-\hat{c} \dot{\beta}_{h}(t)+\hat{q}_{1} \sin (\omega t+m) \cos \left(\beta_{h}(t)\right)\right] d t
$$

By using the symmetry condition and after some simplifications we get

$$
M(m)=-2 \hat{c} \int_{0}^{\infty} \dot{\beta}_{h}^{2}(t) d t+2 \hat{q}_{1} \cos (m) \int_{0}^{\infty} \dot{\beta}_{h}(t) \sin (\omega t) \cos \left(\beta_{h}(t)\right) d t
$$

The first integral, which is always positive, can be rewritten in the form

$$
F_{1}(p, q, \alpha)=\int_{0}^{\infty} \dot{\beta}_{h}^{2}(t) d t=\int_{\beta_{0}}^{\beta_{s}} \dot{\beta}_{h}(\beta) d \beta=\int_{\beta_{0}}^{\beta_{s}} \sqrt{2\left[V\left(\beta_{s}\right)-V(\beta)\right]} d \beta,
$$

which shows that it is just (half) the area inside the unperturbed homoclinic loop (see Eq. (18) and Fig. 7 in Part I). It is the value which has been used therein in the computations of the GIM (see Figs. 8 and 9).

The second integral

$$
F_{2}(\omega, p, q, \alpha)=\int_{0}^{\infty} \dot{\beta}_{h}(t) \sin (\omega t) \cos \left(\beta_{h}(t)\right) d t
$$

represents the Fourier transform of the function $\dot{\beta}_{h}(t) \cos \left(\beta_{h}(t)\right)$; care must be used in its numerical computation. The Melnikov function is then

$$
M(m)=-2 \hat{c} F_{1}+2 \hat{q}_{1} \cos (m) F_{2} .
$$


We have manifolds intersection (and the associated fractalization of the basin of attraction) if there exists an $m$ such that $M(m)=0$ and $M^{\prime}(m) \neq 0$. This is possible if and only if

$$
\frac{\hat{c}}{\hat{q}_{1}}<\frac{\left|F_{2}\right|}{F_{1}},
$$

or, equivalently,

$$
\frac{c}{q_{1}}<\frac{\left|F_{2}\right|}{F_{1}} \stackrel{\text { def }}{=} f(\omega, p, q, \alpha)
$$

an inequality which confirms that we need small damping and/or large excitation amplitude to have complex behaviour. The equality in (22) corresponds to the homoclinic bifurcation threshold triggering the fractalization of basins boundaries, and thus triggering the erosion of the basin of attraction of the periodic orbit close to the rest position.

For $\alpha=0.8$ and $q=0.01$ (providing $p_{K}=0.301$, see Tab. 1 of Part I) the function $f(\omega)$ is reported in Fig. 15 for various values of $p$. The region below $f(\omega)$ is where fractal basin boundaries do occur, while in the region above the critical curve the homoclinic intersection and the related chaotic behaviour is prevented. In the former case it is expected to observe differences between $I F$ and GIM, while in the latter case the differences should be minor.

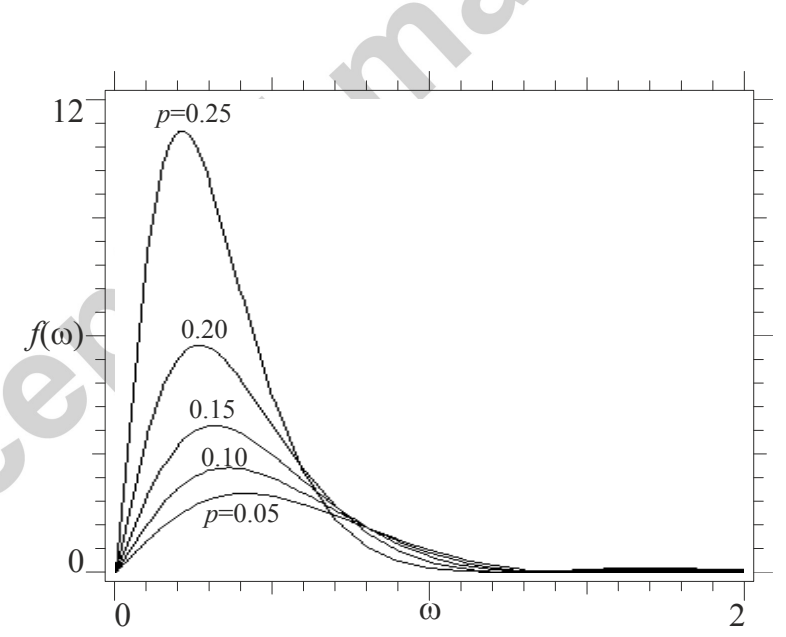

Fig. 15. The function $f(\omega)$ for $\alpha=0.8, q=0.01$ and for different values of $p$. Note that $p_{K}=0.301$.

The main feature highlighted by Fig. 15 is that when $p$ approaches the critical threshold $p_{K}$, the 'chaotic' area (below the curves) increases very much, thus facilitating the fractalization of basin boundaries and the associated reduction of dynamical integrity. This proves why the Thompson load carrying capacity is commonly much lower than the Koiter load carrying capacity.

The function $f(\omega)$ has a sequences of zeros (the first one is scarcely visible - due to the scale of the picture - in Fig. 15 in the interval $\omega=1.2 \div 1.3$ ), meaning that there are also very narrow ranges of 'anti-chaotic' frequencies in between successive regions of 'chaotic' frequencies. Fig. 15 clearly 
shows that the first chaotic region is the most important, while the higher ones are negligible from a practical point of view. Unfortunately, this region $(\omega \in[0,1.2 \div 1.3])$ overlaps with the resonance region $(\omega \in[0,0.623]$, see Fig. 1$)$, so that there is an interaction between resonance and fractalization, which is one further motivation to expect a considerable decrease of the (Thompson) load carrying capacity of the structure also for the two resonant cases not considered in Sect. 4.

The effectiveness of the Melnikov predictions, which is a perturbative technique valid only for small values of $\varepsilon$, has to be checked with computer simulation results. This is done in Fig. 16 just for a case of resonant excitation frequency $(\omega=0.5)$ occurring well below the Koiter threshold. The analysis of the manifolds behaviour as obtained numerically before the theoretically predicted global bifurcation (Fig. 16a), at the bifurcation (Fig. 16b) and after the bifurcation (Fig. 16c), shows just the expected outcomes and fully supports the reliability of the Melnikov asymptotic predictions.

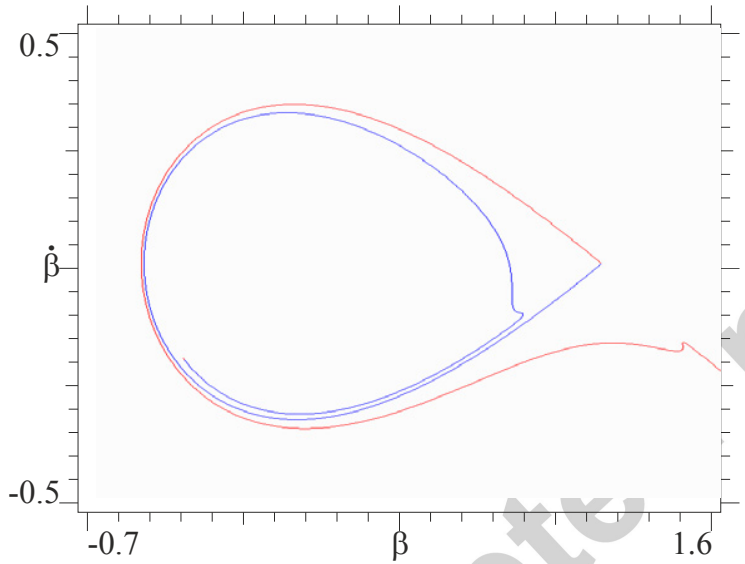

(a)

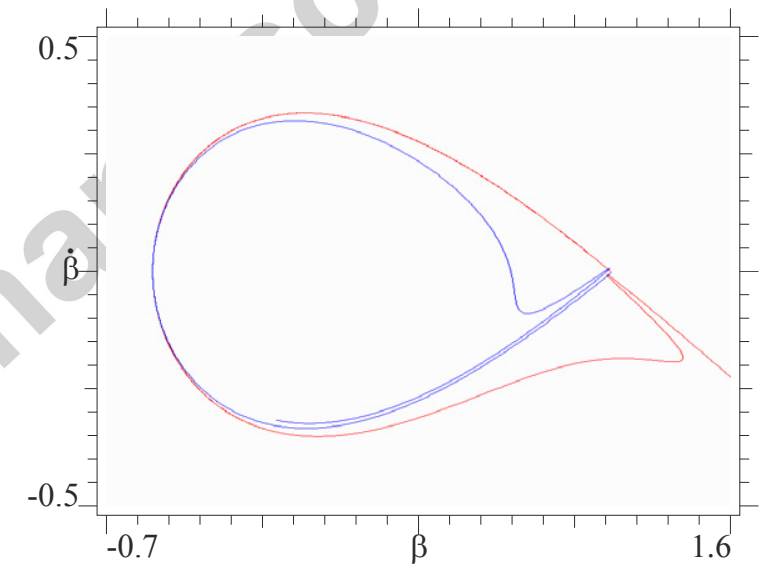

(b)

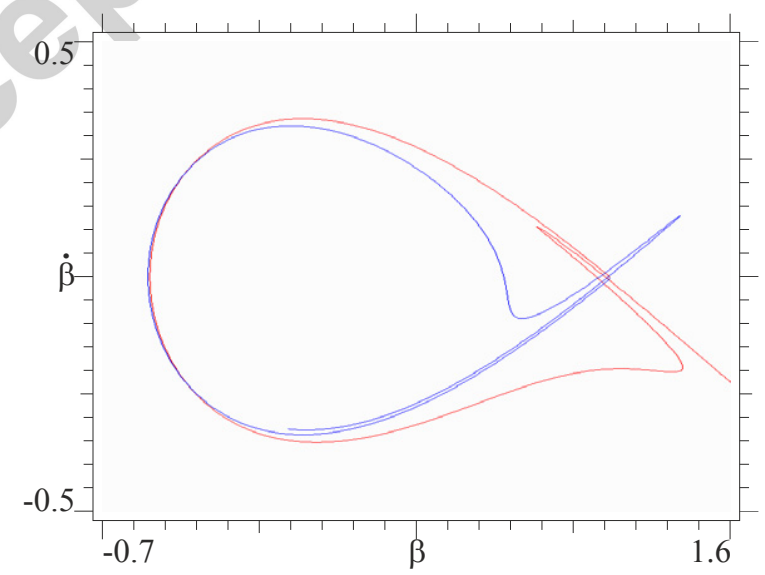

(c)

Fig. 16. (color online) The stable and unstable manifolds behaviour for $\alpha=0.8, q=0.01, p=0.10, \omega=0.5$ (so that $f(\omega)=$ 2.391) and $c=0.01$. (a) $q_{1}=0.003<q_{1, c r}=0.00418$; (b) $q_{1}=0.0042 \cong q_{1, c r}$ and (c) $q_{1}=0.005>q_{1, c r}$. Note that $\omega=0.5$ is close to the resonant value $\Omega=0.532$. 


\section{CONCLUSIONS}

Referring to a simple archetypal mechanical model from the stability theory, the global safety of structures subjected to dynamic excitations has been addressed with the aim of showing that, for practical stability, robust and integer basins are needed.

First, the attractors scenario has been determined, in the case of external non-resonant or resonant excitation, by means of a path following algorithm allowing us to identify the snap or bifurcation points responsible for the loss of stability (in classical sense) of the small oscillations ensuing from the rest position. In the non-resonant case, an approximate analytical solution has been obtained by means of the harmonic balance method, which permits to detect both the saddlenode and the period-doubling bifurcations. In the resonant case, on the other hand, a completely numerical analysis has been performed in the cases of non-interacting or interacting resonance and critical load, the latter case being of course the most involved and the most dangerous from a practical point of view.

Next, the dynamical integrity of the basins of attraction has been investigated. First, it has been shown how the presence of dynamic excitation induces, for certain value of the control parameters, fractal basin boundaries and thus very complicated basins topologies. This entails the so-called erosion of the safe basin, which practically consists in a strong reduction of its compact part without an equivalent reduction of its magnitude (in fact, most of the area remains in the fractal zones, thus not contributing to the safety of the attractor). The non-trivial issue of measuring the integrity, which requires the use of different measures with respect to the sole one used in Part I, has been also discussed. Two different integrity measures previously proposed in the literature have been used.

The integrity profiles, i.e. the integrity measure as a function of a varying parameter, have then been determined, both for increasing static axial load and for increasing dynamic excitation; the former are substantially profiles of attractor robustness, the latter represent actual erosion profiles. Robustness profiles in the presence of dynamic excitation show that the practical (Thompson) critical threshold is lower, and possibly much lower, than the Koiter one, thus extending to the case of periodic attractors the general conclusion drawn in Part I in terms of robustness of the stable equilibria solely occurring in the absence of external excitation. In turn, the erosion profiles highlight how the dynamical integrity of the system close to the Koiter load carrying capacity is merely residual, so that to have a safe behavior a lower, and possibly much lower, practical (Thompson) load carrying capacity must be considered in applications.

As expected, it has also been found that the larger the amplitude of dynamic excitation, the smaller the Thompson load. This highlights the difference, and the interaction, between actual dynamic excitation (time varying force acting on the system) and mere dynamic perturbations (i.e. finite changes in initial conditions), a fact that complicates the global safety analysis. 
As already said, the results of the present paper do confirm and complete those of the companion paper [1], fully accounting for the effects of actual dynamic excitations. The general conclusion to be drawn from the double paper is that, for a safe system design, the robustness and the integrity of its attractors/basins must be properly addressed via global analyses and extensive numerical simulations; a circumstance that is currently overlooked in engineering design, as well as in code prescriptions.

It is the authors' opinion that the global approach is the last theoretical step needed for a full understanding of the load carrying capacity of structures. Now the challenge stands in properly evaluating the dynamical integrity of real structures, where more degrees of freedom may play a role, thus requiring the determination of safe basins in dimensions higher than 2. This is computationally very expensive, and calls for new or improved numerical tools.

At the same time, an open problem is the determination of the acceptable level of residual robustness/integrity, which is required for the effective computation of the Thompson load carrying capacity. This requires a lot of further studies and experiments as well as, possibly, a stochastic approach to the whole matter.

\section{REFERENCES}

[1] S. Lenci, G. Rega, "Load carrying capacity of systems within a global safety perspective. Part I: Robustness of stable equilibria under imperfections." submitted.

[2] L. Euler, Methodus inveniendi lineas curvas maximi minimive proprietate gaudentes, sive solutio problematis isoperimetrici latissimo sensu accepti. Addentamentum 1: de curvis elasticis, Laussanae et Genevae, Apud Marcum-Michaelem, Bousquet et Socios, pp. 245-310, 1744.

[3] W.T. Koiter, 1945, Over de stabiliteit van het elastisch evenwicht, Ph.D. Thesis, Delft University, 1945. English translation: W.T. Koiter, On the Stability of Elastic Equilibrium, NASA Technical Translation F-10, 833, Clearinghouse, US Dept. of Commerce/Nat. Bur. of Standards N67-25033, 1967.

[4] J.M.T. Thompson, "Chaotic phenomena triggering the escape from a potential well", Proceedings of the Royal Society of London A, vol. 421, pp. 195-225, 1989.

[5] M.S. Soliman, J.M.T. Thompson, "Integrity measures quantifying the erosion of smooth and fractal basins of attraction", J. Sound Vibr., vol. 135, pp. 453-475, 1989.

[6] A.N. Lansbury, J.M.T. Thompson, H.B. Stewart, "Basin erosion in the twin-well Duffing oscillator: Two distinct bifurcation scenarios," Int. J. Bif. Chaos, vol. 2, pp. 505-532, 1992.

[7] G. Rega, and S. Lenci, "Dynamical integrity and control of nonlinear mechanical oscillators," J. Vibr. Control, vol. 14, pp. 159-179, 2008.

[8] G. Rega, and S. Lenci, "Identifying, evaluating, and controlling dynamical integrity measures in nonlinear mechanical oscillators," Nonlin. Anal. T. M. \& A., vol. 63, pp. 902-914, 2005.

[9] S. Lenci, G. Rega, "Competing dynamic solutions in a parametrically excited pendulum: attractor robustness and basin integrity," ASME J. Comp. Nonlin. Dyn., vol. 3(4), pp. 41010-, 2008.

[10] P.B. Gonçalves, F.M.A. Silva, G. Rega, S. Lenci, "Global dynamics and integrity of a two-dof model of a parametrically excited cylindrical shell," Nonlin. Dyn., DOI: 10.1007/s11071-010-9785-4.

[11] J. Guckenheimer, P. Holmes, Nonlinear Oscillations, Dynamical Systems and Bifurcation of Vector Fields, Springer-Verlag, New York, 1983.

[12] S. Wiggins, Global Bifurcation and Chaos: Analytical Methods, Springer-Verlag, New York, Heidelberg, Berlin, 1988. 
In this paper the dynamical integrity ideas have been applied to the problem of load carrying capacity of structures.

While the examples refer to an archetypal problem, the underlying ideas are very general

It is believed that they can applied in many applications, even far away from the engineering context. 